Man and Nature

L'homme et la nature

\title{
Les Lumières en Amérique espagnole : plan d'études pour la Colombie coloniale de Moreno y Escandón (1774-1779)
}

Jean-Marie Loncol

Volume 2, 1984

URI : https://id.erudit.org/iderudit/1011817ar

DOI : https://doi.org/10.7202/1011817ar

Aller au sommaire du numéro

Éditeur(s)

Canadian Society for Eighteenth-Century Studies / Société canadienne d'étude du dix-huitième siècle

ISSN

0824-3298 (imprimé)

1927-8810 (numérique)

Découvrir la revue

Citer cet article

Loncol, J.-M. (1984). Les Lumières en Amérique espagnole : plan d'études pour la Colombie coloniale de Moreno y Escandón (1774-1779). Man and Nature / L'homme et la nature, 2, 121-126. https://doi.org/10.7202/1011817ar

\section{Résumé de l'article}

Moreno was not vehemently anti-clerical, as some historians have portrayed him. His plan for the reform of colonial education, summarized in this article, was actually very cautious. It failed primarily because of opposition from the Dominicans.
Copyright (C Canadian Society for Eighteenth-Century Studies / Sociéte canadienne d'étude du dix-huitième siècle, 1984
Ce document est protégé par la loi sur le droit d'auteur. L'utilisation des services d'Érudit (y compris la reproduction) est assujettie à sa politique d'utilisation que vous pouvez consulter en ligne.

https://apropos.erudit.org/fr/usagers/politique-dutilisation/ 
LES LUMIERES EN AMERIQUE ESPAGNOLE: PLAN D'ETUDES POUR

LA COLOMBIE COLONIALE DE MORENO Y ESCANDON (1774-1779)

Jean-Marie Loncol

Quel impact a connu vraiment la Philosophie des Lumières dans le monde hispanique? Pour ne pas nous ramener à la vieille légende noire, pareille question exigerait sans doute une réponse extrêmement nuancée à partir de recherches précises qu'orienterait une problématique renouvelée. Les études socio-économiques ainsi que socio-politiques récentes et en cours dicteraient enfin des approches fécondes. La présente communication, loin de prétendre à pareil objectif, rappelle simplement les obstacles évoqués pour les adeptes de cette Philosophie des Lumières par Marcelin Defourneaux, entre autres.l Nous reconnaissons aussi l'échec partiel déploré notamment par Jean Sarrailh. ${ }^{2}$ Et, rencontrant le problème dans nos analyses d'histoire hispano-américaine pour la seconde moitié du XVIIIe siècle, nous voudrions apporter aux ouvrages des auteurs mentionnés une modeste confirmation quant à l'essentiel mais aussi quelques nuances ou précisions utiles, concernant les causes et la nature du demi-échec, en résumant l'épisode du projet d'études que soumettait Moreno y Escandón pour la Nouvelle-Grenade en 1774 .

Le haut fonctionnaire dont les options pédagogiques vont nous occuper ici a fait l'objet d'une première présentation lors de notre congrès de London en mai 1980. Nous nous disposions alors à suivre en détail toute la carrière de ce Francisco Antonio Moreno y Dlaz de Escandón à travers la vice-royauté de Nouvelle-Grenade, la vice-royauté du Pérou et la capitainerie générale du Chili entre les années 1760 et le décès du personnage en 1792. Il nous a fallu depuis cet essai préliminaire rectifier certaines hypothèses en examinant les activités de Moreno aux archives de Séville. 
A moins que des dossiers importants ne nous aient échappé en cette première étape, et jusqu'à ce qu'il nous soit possible de compléter la recherche quelque jour peut-être à Santiago du Chili, les préoccupations didactiques de Don Francisco Antonio ne nous semblent pas s'être exprimées clairement en dehors de la Nouvelle-Grenade. A quel titre et pour quelles raisons s'est-il intéressé aux études là-bas? Dans quel contexte a-t-il élaboré son programme? Fils d'Espagnols européens, Moreno est né en Amérique, dans la ville colombienne de Mariquita. Il a obtenu son doctorat en théologie et en droit, puis entrepris une carrière de professeur de droit canon, au Collège royal supérieur de San Bartolomé portant aussi le nom d'Université de San Javier, sous l'autorité des Jésuites dans la capitale de Santa $\mathrm{Fe}$ de Bogotá. Notre homme est devenu surtout, à l'Audience de Santa Fe, protecteur des Indiens et Fiscal, c'est-à-dire procureur du Roi. Ce dernier poste lui confiait la surveillance officielle de l'enseignement.

Le facteur décisif dans la genèse du plan qui nous intéresse est l'expulsion des Jésuites ordonnée par Charles III en 1767 et qui secoue la machine de l'enseignement ici comme dans le reste de l'Amérique espagnole. Quel était alors l'essentiel de cette machine aux niveaux secondaire et supérieur en Nouvelle-Grenade? Outre les collèges et séminaires qui s'étaient implantés aux XVIe et XVIIe siècles ainsi que durant les premières décennies du XVIIIe siècle dans les villes les plus importantes comme Cartagena, Tunja, Popayán, deux institutions de haut savoir avaient surgi dans la capitale. Ces institutions avaient mené entre elles, pour obtenir le statut d'université, la lutte classique opposant les communautés religieuses. Il s'agit de Santo Tomás pour les Dominicains et de San Javier dont il a déjà été question pour les Jésuites. Quelles sortes de cours y dispensait-on? Si l'on schématise un peu ici, guidé par des ouvrages généraux, 3 on voit que--le but fondamental de l'instruction publique étant encore une meilleure connaissance de Dieu par l'analyse de sa création afin de mieux atteindre le salut éternel--la formation générale de la jeunesse se ramenait au trivium (grammaire, dialectique, rhétorique) et au cuatrivium (musique, arithmétique, géométrie, astronomie) tandis que les études supérieures, ouvrant pour l'essentiel sur les activités ecclésiastiques et légales, s'occupaient principalement de philosophie et de théologie, de droit canon et de droit civil.

A partir de 1767, les autorités métropolitaines voudraient profiter de la disparition du magistère jésuitique pour réformer en profondeur les études secondaires et lancer vraiment les études universitaires en NouvelleGrenade comme dans les autres possessions de Sa Majesté Catholique. Pour ce faire elles ont été influencées dans une certaine mesure par les essais critiques de Benito Jerónimo Feijoo, ce bien modéré champion espagnol des Lumières dont les principales publications s'échelonnent de 1727 à 1760 . Il n'est pas question au point de vue universitaire, dans ce contexte, d'accorder aux moines de Saint-Dominique la place laissée vacante à Santa Fe par l'expulsion de la Compagnie de Jésus. L'Université de Santo Tomas perd le pouvoir de décerner les grades supérieurs et est réduite au niveau d'un collège secondaire. A sa place, et pour remplacer aussi l'ancienne institution des Jésuites, on désire instaurer une université dite publique qui releverait directement du monarque sans que celui-ci ait à passer par le chapitre ou le supérieur, même docile, d'un ordre religieux quelconque. Le nom de Moreno y Escandon est lié à ce projet. Don Francisco Antonio 
aurait-il été l'anticlérical habile et obstiné que des historiens colombiens ${ }^{4}$ fustigeront par la suite? Cet ancien élève et employé des Jésuites se serait-il acharné, comme on l'a laissé entendre, 5 sur les dépouilles de la Compagnie mise hors-la-loi pour que triomphent enfin en NouvelleGrenade les seuls critères de la raison? L'objectivité historique nous impose ici, pour le moins, une double mise au point. Tout d'abord la nomination de Moreno à la Junta de Temporalidades, à la Junte du Temporel chargée de disposer au mieux à Santa Fe des biens confisqués aux Jésuites, est liée tout simplement à ses fonctions de procureur du Roi. Et notons d'ailleurs que sa conduite en l'occurence révélera chez lui ce qu'il est convenu d'appeler un esprit éclairé puisqu'il décidera d'appliquer les anciens avoirs des Pères au bien de la collectivité sous la forme d'un nouvel hospice pour vieillards pauvres et d'une bibliothèque publique formant aujourd'hui le noyau de la Bibliothèque nationale colombienne. De même sa participation à la réforme de l'enseignement découle de sa qualité de Fiscal. Membre à ce titre de la Junte d'Etudes présidée par le vice-roi Manuel de Guirior, en compagnie de l'archevêque ainsi que des recteurs des collèges de San Bartolomé et du Rosario, Don Francisco Antonio est nommé par cette junte "directeur royal des Etudes" avec pour mission d'élaborer le projet qui nous occupe à présent. 6

Ce Plan ou Méthode provisoire, 7 soumis le 12 septembre 1774 , ne concerne pas l'université publique souhaitée, précisons-le immédiatement. Le sort fait au programme expliquera bien tout à l'heure la prudence du réformateur ainsi que la lenteur de la réforme. Laissons de côté ici les énoncés de principes, par lesquels commence le texte, sur la nécessité pour le gouvernement de surveiller l'instruction publique, si importante au bien de l'Etat, et sur la nécessité aussi de choisir comme professeurs des personnes honnêtes et vertueuses. Ignorons de même les cours de latin qui relèvent manifestement du niveau secondaire. Seules les branches de la philosophie, de la théologie et du droit peuvent faire à Santa $\mathrm{Fe}$, selon le projet de Don Francisco Antonio, l'objet d'études relativement avancées. Le programme de philosophie, qu'il faut avoir réussi pour aborder les autres disciplines dont nous parlerons plus loin, s'adresse à des élèves de 12,13 ou 14 ans. Il doit durer trois ans de façon à ce que ces élèves puissent atteindre les facultés qualifiées de majeures vers l'âge de 15, 16 ou 17 ans. La première année comprend des éléments de logique--enseignant la façon de bien raisonner--puis des éléments d'arithmétique, d'algèbre, de géométrie et de trigonométrie. En deuxième année vient la physique divisée en physique générale, équivalant à ce que nous appelons vraiment physique, et en physique spéciale qui englobe la géographie du pays, l'histoire naturelle, l'observation météorologique, l'agriculture et la connaissance des métaux précieux. La troisième année, enfin, concerne la métaphysique et se termine par la philosophie morale si nécessaire, estil remarqué, aux théologiens et aux juristes. Nous voici parvenus aux études vraiment supérieures qui ne touchent donc que la théologie et le droit.

Afin de couper aux énumérations ennuyeuses et inutiles en ce qui concerne notre point de vue profane, retenons simplement du programme de théologie la prépondérance accordée au contact direct avec l'Ecriture sainte ainsi qu'à la mémorisation d'une partie au moins de ce long corpus. En première année, les 2.528 vers du Psautier doivent être retenus tandis que les 909 chapitres de l'Ancien Testament doivent être lus à raison de trois 
chapitres par soir. En deuxième année, les deux évangiles de Saint-Mathieu et de Saint-Marc ainsi que les huit premiers chapitres de celui de SaintLuc seront appris par coeur. Il faudra, en troisième année, compléter de la même façon l'évangile de Saint-Luc et y ajouter celui de Saint-Jean avec les quinze premiers chapitre des Actes des Apôtres. Viendront, en quatrième année, le reste de ces Actes et les six premières épitres de Saint-Paul. Les huit dernières épitres de Saint-Paul seront apprises en cinquième année avec celle de Saint-Jacques, les deux de Saint-Pierre, les trois de Saint-Jean, celle de Saint-Jude et finalement l'Apocalypse. $\mathrm{Pa}-$ rallèlement à cette étude doit se faire, tout au long de ces cinq années, la lecture des Ecrits conciliaires ainsi que des oeuvres des Pères de l'Eglise.

En droit, où les cinq années du cours répondent à une ordonnance royale de 1771, une certaine progression pédagogique est stipulée par notre plan. Les professeurs choisiront en effet une ou deux rubriques des Decretales du juriste espagnol Gregorio Nono et les expliqueront toute une année sans donner d'abord aux étudiants les définitions et les distinctions en rapport avec le droit canon, les compilations et les sources dont la connaissance, souligne-t-on, est pourtant indispensable aux juges ecclésiastiques et laics. Les données requises à ce propos ne seront fournies aux étudiants que peu à peu. Il est impossible d'autre part, estime le texte, de séparer vraiment droit canonique et droit civil. Il importe au contraire de les unir dans un même programme pour que les étudiants puissent en retirer un profit d'ensemble. Pareille intégration ne commencera pas cependant dès le début des études. Les deux premières années devront faire connaitre d'abord l'histoire du droit romain à partir des quatre livres des Instituta de Justinien selon le principe de deux livres par an. On démontrera aussi en parallèle la concordance des lois de Sa Majesté Catholique. C'est en troisième année que commencera l'étude du droit canonique avec une analyse de ses origines, de ses sources et de son autorité. La même chose vaudra pour les conciles de l'Eglise. Un examen spécial sera fait alors de la participation aux conciles américains du monarque espagnol avec son droit universel et absolu relativement à la propagation de la Foi dans le Nouveau Monde. Les quatrième et cinquième années, enfin, seront consacrées aux institutions canoniques selon la méthode suivie à l'Université espagnole d'Alcalá. Mentionnons, pour conclure ce projet, l'absence de la médecine qui faisait pourtant l'objet d'une série de cours en 1774 au collège du Rosario. Sans que nous puissions juger du mérite de la décision, cet enseignement est maintenant interrompu jusqu'à ce que le programme, dûment réformé, puisse obtenir une sanction officielle.

Le plan d'études de Moreno y Escandón, dont nous venons de résumer les grandes lignes, ne parait pas devoir bouleverser l'édifice culturel de la Nouvelle-Grenade. Encore faudrait-il examiner attentivement toutes les parties de cet édifice et confronter le projet dans ses menus détails à la routine pédagogique alors en vigueur là-bas. Le programme de Don Francisco Antonio est mis en application séance tenante, de manière provisoire et jusqu'à ce que le Roi en décide autrement, sur ordre du vice-roi Manuel de Guirior. La réaction ne se fait pas attendre, cependant. Les Dominicains, voyant leur échapper le monopole de l'enseignement supérieur que la disparition des Jésuites semblait devoir leur promettre pourtant, interviennent vite directement et indirectement de multiples façons. Bornons-nous à 
mentionner ici la critique du 21 juin 1779 adressée au vice-roi--Manuel Antonio Flórez alors--par l'auditeur hostile à Moreno qu'était de manière générale Joaquín Vasco y Vargas. ${ }^{8}$ Les griefs élevés devant le pouvoir colonial et devant le pouvoir métropolitain contre le programme de 1774 obligent à Santa $\mathrm{Fe}$ la Junte des Etudes--Don Francisco Antonio en faisant toujours partie--à remanier le projet pour suivre davantage la tradition comme le désire Charles III. Les remaniements sont opérés en octobre 1779. Ils sont jugés insuffisants néanmoins. La hargne des rebelles comuneros envers Moreno, exprimée surtout dans le placard qui apparait sur un mur de la capitale le 7 avril 1781 , se rapporte à l'inspection des terres indigènes faite par le protecteur des Indiens en 1777-78. Elle vise aussi et surtout l'offensive sournoise qu'aurait menée notre homme depuis 1774 contre la sainte religion par le biais de son programme d'études.9

Muté à Lima dès 1780 et décédé à Santiago du Chili en 1792 comme nous le signalions, Don Francisco Antonio va en fin de compte perdre la partie au profit des Dominicains de façon posthume, pourrait-on dire. L'épilogue de notre point d'histoire se fera en effet attendre jusqu'à la fin du siècle. Le sursaut de conservatisme provoqué en Espagne par la Révolution française l'emporte alors sur le réformisme relativement modéré qui animait un fonctionnaire comme celui dont nous venons de parler. Une dernière requête est adressée à Charles IV par le recteur de l'ancienne université dominicaine de Santa Fe. Soumise au Conseil des Indes le 16 juin 1797, elle reçoit en définitive une réponse favorable. Par une cédule royale du 27 octobre 1798, l'Université de Santo Tomás retrouve ses privilèges académiques et le plan d'études de Moreno y Escandon est finalement désavoué.10 Faut-il voir ici un triomphe total de la réaction? L'Espagne noire aurait-elle eu raison de l'Espagne rouge en Amérique? Nous savons bien que la science fuit de telles simplifications. Les Lumières envisagées par notre "directeur royal des Etudes" n'ont pu triompher en NouvelleGrenade à la fin du XVIIIe siècle pour diverses raisons qu'expliquerait pour l'essentiel la crise vécue alors par l'ensemble du monde occidental. Mais I'Histoire continue...

Université de Montréal

NOTES

1 Marcelin Defourneaux, Pablo de Olavide ou l'Afrancesado (1725-1803) (Paris: P.U.F., 1959).

2 Jean Sarrailh, L'Espagne éclairée de la seconde moitié du XVIIIe siècle (Paris: Librairie C. Klincksieck, 1964).

3 Par exemple ceux du P. Fr. José Abel Salazar, Los Estudios eclesiásticos en el Nuevo Reino de Granada (1563-1810) (Madrid: C.S.I.C., 1946) et de Gabriel Porras Troconis, Historia de la Cultura en el Nuevo Reino de Granade (Sevilla: E.E.H.A., 1952). 
4 Notamment José Manuel Groot, Historia Eclesiástica y Civil de Nueva Granada (Bogota, 1889), II, p. 164 .

5 Sergio Elías Ortiz, Nuevo Reino de Grenada. El Virreynato (17531810), vol. IV, t. 2 de Historia extensa de Colombia (Bogota, 1970), p. 141 .

6 E. Posada et P.M. Ibáñez, ed., Relaciones de Mando. Memorias presentadas por los gobernantes del Nuevo Reino de Granada (Bogota, 1910), p. 158.

7 Archivo General de Indias, Séville (à l'avenir: A.G.I.), Santa Fe, leg. 759.

8 A.G.I., Santa Fe, leg. 736-B.

9 A.G.I., Santa Fe, leg. 663-A.

10 A.G.I., Santa Fe, leg. 759. 